\title{
Relativistic Spacetime Structure
}

\author{
Samuel C. Fletcher* \\ Department of Philosophy \\ University of Minnesota, Twin Cities \\ $\mathcal{E}$ \\ Munich Center for Mathematical Philosophy \\ Ludwig Maximilian University of Munich
}

August 12, 2019

\begin{abstract}
I survey from a modern perspective what spacetime structure there is according to the general theory of relativity, and what of it determines what else. I describe in some detail both the "standard" and various alternative answers to these questions. Besides bringing many underexplored topics to the attention of philosophers of physics and of science, metaphysicians of science, and foundationally minded physicists, I also aim to cast other, more familiar ones in a new light.
\end{abstract}

\section{Introduction and Scope}

In the broadest sense, spacetime structure consists in the totality of relations between events and processes described in a spacetime theory, including distance, duration, motion, and (more generally) change. A spacetime theory can attribute more or less such structure, and some parts of that structure may determine other parts. The nature of these structures and their relations of determination bear on the interpretation of the theory-what the world would be like if the theory were true (North, 2009). For example, the structures of spacetime might be taken as its ontological or conceptual posits, and the determination relations might indicate which of these structures is more fundamental (North, 2018). Different perspectives on these questions might also reveal structural similarities with other spacetime theories, providing the resources to articulate how the picture of the world that that theory provides is different (if at all) from what came before, and might be different from what is yet to come. ${ }^{1}$

*Juliusz Doboszewski, Laurenz Hudetz, Eleanor Knox, J. B. Manchak, James Read, and Gergely Székely have my gratitude for comments on earlier drafts.

${ }^{1}$ For example, it might do so by showing that one spacetime theory posits less structure than another (Barrett, 2015). If there is a relevant sense in which the theories are nevertheless equivalent, then this structural surplus might be deemed superfluous (Weatherall, 2017). 
My present twofold goal is to survey (in sections 2 and 3) in particular the spacetime structures of the general theory of relativity - our best current theory of space, time, and gravitation - and (in sections 4 and 5) the determination relations between them, including both what I take the "standard" account of these to be (in sections 2 and 4) and alternative perspectives worth investigating (in sections 3 and 5). ${ }^{2}$ Along the way, many conceptual and technical questions arise that are worth further research. Some of these questions have been discussed extensively in the physics and philosophy literatures, some less so. ${ }^{3}$ My hope is that they will stimulate readers' interests to pursue them.

\section{What There Is: The Standard Account}

The standard account of relativistic spacetime structure divides that structure into two classes: the manifold structure of events and the geometrical structure built upon it. This division does not entail that the two types of structures must be interpreted as ontologically independent (Pooley, 2006). Nevertheless it will aid exposition to introduce these two types of structures separately; one only has to keep in mind that the interpretation of some of these structures as they are introducedin particular, those of the manifold in section 2.1—may be incomplete until others are introducedin particular, those of the geometry in section 2.2.

\subsection{Standard Manifold Structure}

The events of a relativistic spacetime consist in an uncountable set $M$; they represent all the potential or actual point-sized happenings - that is, without spatial or temporal extension-everywhere and everywhen in that spacetime. Consequently, collections of events represent processes, histories of particular objects, and so on. But, not all collections of events do so: first, one may intend to use the same relativistic spacetime model to describe different states of affairs, in which happenings occur at different points relative to one another; second, one typically restricts attention to continuously connected events to describe processes and histories.

To distinguish between continuous and discontinuous collections of events, one must use the topological structure of the spacetime's events. In particular, the events $M$ are equipped with a topology $\mathcal{T}$ that makes them into a topological four-manifold (Lee, 2011). Most simply, a topological four-manifold is a topological space that is locally homeomorphic to $\mathbb{R}^{4}$ with its usual topology: for every point $p \in M$, there is a homeomorphism $\varphi: U \rightarrow V$, where $p \in U \in \mathcal{T}$ and $V$ is an open subset of $\mathbb{R}^{4}$. Thus a neighborhood of every event—some collection of events spatiotemporally proximal to it - can be described with four coordinates, anticipating their interpretation as three spatial and one temporal coordinate. ${ }^{4}$

\footnotetext{
${ }^{2}$ For lack of space, I assume some familiarity with topological manifolds (Lee, 2011), differential geometry (Kobayashi and Nomizu, 1963, 1969), and the abstract index notation used in mathematical relativity (Wald, 1984; Malament, 2012) but have also endeavored to provide references where the elements I discuss may be studied in more detail, in particular Hawking and Ellis (1973).

${ }^{3}$ The approaches most similar to mine are perhaps Ehlers (1973), and Torretti (1996, Ch. 6.1); for a more technical but elegant presentation, see Trautman (1976).

${ }^{4}$ I have not yet introduced the geometrical structure needed to support these interpretations; nevertheless, one might demand four-dimensionality from empirical observation or perhaps even from a priori considerations on the form of intuition (Carnap, 1922).
} 
The events' topology is required to satisfy several further conditions (Geroch and Horowitz, 1979, p. 218). First, it is Hausdorff, meaning that if two points of $M$ are distinct, then there are neighborhoods of each that are disjoint. This is really an extension of the idea that, locally, events should have the continuous structure of a region of $\mathbb{R}^{4}$. Second, it is path-connected, meaning that for every $p, q \in M$, there is a continuous function $f:[0,1] \rightarrow M$ such that $f(0)=p$ and $f(1)=q .^{5}$ If there were events not so connectible, they would not bear any spatiotemporal relations to each other at all, and could scarcely be said to be part of the same universe. ${ }^{6}$ Third, it is secondcountable: there is a countable base for $\mathcal{T}$, meaning that there is a countable subset $\tau \subset \mathcal{T}$ such that every open set in $\mathcal{T}$ can be expressed as the union of elements from $\tau$. This condition prevents the collection of events from being too "large" or "spread out," and is equivalent (when conjoined with the Hausdorff condition) to the manifold being embeddable in a finite-dimensional Euclidean space. ${ }^{7}$

The topological structure $T$ of a spacetime provides the sense in which certain sets $S$ of events are continuously connected - those for which there is a path between every pair of elements in $S$. But it does not provide the structure needed to describe the directions of paths (i.e., tangent vectors), their rates of change, and so on. For these concepts, one needs to be able to do calculus on the manifold, which is provided by a differentiable structure. It will be convenient to define this structure in terms of (four-)charts, versions of which we have already encountered in expressing the sense in which a topological four-manifold is locally $\mathbb{R}^{4}$.

A (four-)chart on $M$ is an injective map $\varphi: U \rightarrow \mathbb{R}^{4}$, where $U \subseteq M$ and $\varphi[U]$ is open in $\mathbb{R}^{4}$. An atlas $\mathcal{A}$ for $M$ is a collection of charts whose domains cover $M$. Finally, a $C^{k}$ differentiable structure on $M$ (for $k \geq 1$ ) is an atlas whose charts are mutually $C^{k}$ compatible: for every $\phi, \phi^{\prime} \in \mathcal{A}$ with respective domains $U, U^{\prime} \subseteq M$, if $U \cap U^{\prime} \neq \emptyset$, then $\phi \circ \phi^{-1}: \phi^{\prime}\left[U \cap U^{\prime}\right] \rightarrow \phi\left[U \cap U^{\prime}\right]$ is a $C^{k}$ function-i.e, $k$ times continuously differentiable-from an open set of $\mathbb{R}^{4}$ to an open set of $\mathbb{R}^{4}$. (Such compositions are often called transition maps or changes of coordinates.) A differential structure that is as large as it can be is called maximal: it contains all the charts that are mutually compatible.

The differential structure of spacetime events is standardly required to be smooth $\left(C^{\infty}\right)$, maximal, and compatible with its topological structure $\mathcal{T}$ in the following sense: the domain of every chart in $\mathcal{A}$ is an open set in $\mathcal{T}$. This compatibility condition ensures that the continuous and differentiable structures mesh with one another, in that the transition maps of the latter are also continuous with respect to the former. The maximality condition is also extremely important, for it ensures that there are no distinguished coordinate systems: each one that is compatible is on an equal footing to describe locally a collection of events. This is, in one sense, what characterizes manifolds as geometrical objects. Finally the smoothness $\left(C^{\infty}\right)$ condition provides the structure to describe arbitrary orders of derivatives of quantities or fields, which, consonant with the maximality of the differentiable structure, does not depend on particular coordinate systems.

In sum, the standard manifold structure of a spacetime provides the means to describe how collections of events form continuous or even smooth collections by constraining them to be locally

\footnotetext{
${ }^{5}$ One usually finds the requirement stated that as a topological space $M$ must connected rather than pathconnected-i.e., that the only clopen sets are $M$ and $\emptyset$-but these two conditions are equivalent for manifolds and being path-connected has a more obvious interpretation.

${ }^{6} \mathrm{Cf}$. the arguments of Lewis (1986) on how to separate worlds in the context of defending his modal realism.

${ }^{7}$ For Hausdorff connected manifolds, being second-countable is also equivalent to being paracompact, being Lindelöf, and the global existence of a covariant derivative operator-see section 4.
} 
like $\mathbb{R}^{4}$. But this structure does not yet do so for concepts of temporal duration, spatial extension, and motion, whether straight or curvilinear. For these one must add geometrical structure.

\subsection{Standard Geometrical Structure}

The covariant derivative operator (or affine connection) $\nabla$ of a relativistic spacetime provides a standard of constancy for general tensor fields on that spacetime. In particular, it allows one to describe a sense in which certain curves are straight, while others are curvilinear.

A derivative operator at a point $p \in M$ is a map from $(q, r)$-tensor fields $\Phi_{b_{1} \cdots b_{r}}^{a_{1} \cdots a_{q}}$ at $p$ to $(q, r+1)$ tensor fields $\nabla_{c} \Phi_{b_{1} \cdots b_{r}}^{a_{1} \cdots a_{q}}$ at $p$ satisfying the following conditions (Hawking and Ellis, 1973, §2.5):

1. It commutes with addition; e.g., $\nabla_{c}\left(\Phi_{b_{1} \cdots b_{r}}^{a_{1} \cdots a_{q}}+\Phi_{b_{1} \cdots b_{r}}^{a_{1} \cdots a_{q}}\right)=\nabla_{c} \Phi_{b_{1} \cdots b_{r}}^{a_{1} \cdots a_{q}}+\nabla_{c} \Phi_{b_{1} \cdots b_{r}}^{a_{1} \cdots a_{q}}$.

2. It satisfies the Leibniz rule for tensor multiplication; e.g., $\nabla_{e}\left(\Phi_{b_{1} \cdots b_{r}}^{a_{1} \cdots a_{q}} \Psi_{d_{1} \cdots d_{t}}^{c_{1} \cdots c_{s}}\right)=\left(\nabla_{e} \Phi_{b_{1} \cdots b_{r}}^{a_{1} \cdots a_{q}}\right) \Psi_{d_{1} \cdots d_{t}}^{c_{1} \cdots c_{s}}+\Phi_{b_{1} \cdots b_{r}}^{a_{1} \cdots a_{q}}\left(\nabla_{e} \Psi_{d_{1} \cdots d_{t}}^{c_{1} \cdots c_{s}}\right)$

3. It commutes with contraction; e.g., contracting the indices $a_{i} \rightarrow b_{j}$ of $\Phi_{b_{1} \cdots b_{r}}^{a_{1} \cdots a_{q}}$ (for any $i \in$ $\{1, \ldots, q\}$ and $j \in\{1, \ldots, r\})$ and then applying $\nabla$ is the same as contracting the indices $a_{i} \rightarrow b_{j}$ of $\nabla_{c} \Phi_{b_{1} \cdots b_{r}}^{a_{1} \cdots a_{q}}$.

4. For any scalar field $f: M \rightarrow \mathbb{R}, \nabla_{a} f=d_{a} f$, where $d$ is the exterior derivative operator.

If $\xi^{a}$ is the tangent vector field to a curve $\gamma: I \rightarrow M$ (where $I$ is any interval of $\mathbb{R}$ ), then at points on the image of $\gamma, \xi^{c} \nabla_{c} \Phi_{b_{1} \cdots b_{r}}^{a_{1} \cdots a_{q}}$ is a directional derivative of the field $\Phi_{b_{1} \cdots b_{r}}^{a_{1} \cdots a_{q}}$ along $\gamma$. In particular, $\xi^{a} \nabla_{b} \xi^{b}$ measures (according to $\nabla$ ) the extent to which $\xi^{b}$ is constant along $\gamma$, is self-parallel or locally straight. It is so constant when $\xi^{a} \nabla_{b} \xi^{b}=\mathbf{0}$ at each point of the image, in which case $\gamma$ is said to be a geodesic. Now, a relativistic spacetime assumes a single, globally defined (i.e., at every $p \in M$ ) derivative operator. The images of geodesics are then interpreted as the (locally) straight lines of events in a spacetime.

It is important to note, however, that the property of being a geodesic depends on parameterization: if $\gamma$ is a geodesic, then for any diffeomorphism $\alpha: I^{\prime} \rightarrow I, \alpha \circ \gamma$ is a geodesic if and only if $\alpha$ is linear. Conversely, if the tangent vector $\xi^{a}$ satisfies the weaker self-parallel condition $\xi^{a} \nabla_{b} \xi^{b}=f \xi^{a}$ for some scalar function $f$ on the image of $\gamma$, then there is always a diffeomorphism $\alpha: I^{\prime} \rightarrow I$ such that $\alpha \circ \gamma$ is a geodesic. Such curves are called pregeodesics; they have the same images as the geodesics, but are less restrictive regarding parameterization. This interpretative importance motivates the definition of a projective structure on spacetime, which is the equivalence class of all affine connections that determine the same pregeodesics-they agree on which collections of points constitute locally straight lines.

Algebraically, the covariant derivative may not commute with itself. Geometrically, in determining which lines are locally straight, the affine connection can allow for initially parallel geodesics to converge or diverge, and to twist. These two features are encoded in the Riemann curvature and torsion tensors, $R_{b c d}^{a}$ and $T_{b c}^{a}$ respectively, which are uniquely defined by their action on vector fields $X^{a}, Y^{b}, Z^{c}$ :

$$
\begin{aligned}
R_{b c d}^{a} X^{b} Y^{c} Z^{d} & =X^{b} \nabla_{b}\left(Y^{c} \nabla_{c} Z^{a}\right)-Y^{c} \nabla_{c}\left(X^{b} \nabla_{b} Z^{a}\right)-[X, Y]^{b} \nabla_{b} Z^{a}, \\
T_{b c}^{a} X^{b} Y^{c} & =X^{b} \nabla_{b} Y^{a}-Y^{b} \nabla_{b} X^{a}-[X, Y]^{a} .
\end{aligned}
$$


Here, $[X, Y]^{b}$ is the commutator (or Lie bracket) between vector fields $X^{a}$ and $Y^{a}$ (Malament, 2012, pp. 44-45). In general relativity, one requires the torsion of the connection to vanish everywhere, but the curvature is general is not fixed. This entails that the derivative operator commutes on scalar fields $f$, i.e., $\nabla_{a} \nabla_{b} f=\nabla_{b} \nabla_{a} f$ (Hawking and Ellis, 1973, p. 34).

The affine connection provides a standard of change for tensor fields defined within copies of the tangent and cotangent spaces at each event. But it does not provide the structure needed to distinguish time and space-i.e., describe durations and distances-or angles of incidence beyond parallelism. In general relativity, the structure that supports these concepts is, essentially, the spacetime metric, which is a smooth, symmetric field $g_{a b}$ that is invertible-i.e., there exists an inverse metric $g^{b c}$ such that $g_{a b} g^{b c}$ is the $(1,1)$-identity tensor-and has Lorentz signature $(1,3)$. Thus, one of the functions of the metric is an inner product structure on the tangent space at a point that assigns between any two non-zero vectors $X^{a}, Y^{a}$ there a "cosine angle" $g_{a b} X^{a} Y^{b} /\left|\left(g_{a b} X^{a} X^{b}\right)\left(g_{a b} Y^{a} Y^{b}\right)\right|^{1 / 2}$, hence a norm structure on the space: $\left\|X^{a}\right\|_{g}=\left|g_{a b} X^{a} X^{b}\right|^{1 / 2}$. Based on this inner product, the elements $X^{a}$ of the tangent space are divided into three classes:

- If $g_{a b} X^{a} X^{b}>0, X^{a}$ is timelike: it represents an instantaneous direction in the manifold of events corresponding to differences in time, hence an instantaneous trajectory for a positivemass particle.

- If $g_{a b} X^{a} X^{b}=0, X^{a}$ is null (or lightlike): it represents an instantaneous direction in the manifold of events corresponding to an instantaneous trajectory for a zero-mass particle, such as a light ray.

- if $g_{a b} X^{a} X^{b}<0, X^{a}$ is spacelike: it represents an instantaneous direction in the manifold of events corresponding to a spatial direction.

In the tangent space, the null vectors form a double cone structure-the light cones-with the apex of the cones at the origin. The vectors inside the cones are precisely the timelike ones, while those outside are spacelike. Causal vectors are said to be those that are either timelike or null. Curves inherit these classifications, so that those with always timelike tangent vectors represent the possible worldlines, or locus of present events, of a positive-mass particle, and similarly null curves represent the possible worldlines of zero-mass particles. Spacelike curves represent, in some sense, spatial paths.

The significance of this classification is more apparent when one considers how the spacetime metric provides an integration measure for assigning magnitudes to continuous (and piecewise differentiable) timelike and spacelike curves. (Null curves are always assigned a magnitude of zero.) If $\gamma:\left[s_{0}, s_{1}\right] \rightarrow M$ is a timelike or spacelike curve with tangent vector $\xi^{a}$, then $\|\gamma\|=$ $\int_{s_{0}}^{s_{1}}\left\|\xi^{a}\right\|_{g} d s$. If $\gamma$ has a unit tangent vector-i.e., $\left\|\xi^{a}\right\|_{g}=1$ - then its magnitude is just $s_{1}-s_{0}$. For spacelike curves, this is just the spatial length of the curve. The magnitude of a timelike curve is interpreted as the duration of the curve, i.e., the time elapsed that a particle with the image of $\gamma$ as its worldline would experience. In general, the magnitude of a curve does not just depend on its endpoints (if it has any), hence it cannot in general be interpreted as a distance function between those points; this is the source of the well-known "twin paradox."

\footnotetext{
${ }^{8}$ One can in certain circumstances define a Lorentzian "distance" function between (timelike- or spacelike-related) events as the infimum of the magnitudes of the (timelike or spacelike) curves between them, but this function does not satisfy the usual requirements of a distance function, nor can it always be globally defined on $M$. Nevertheless, one might still say that there is a local Lorentzian distance structure on the spacetime (Beem et al., 1996, Def. 4.25).
} 
In addition to providing for the structure for durations and lengths, the spacetime metric also does so for hypervolumes - in general, temporally extended volumes - through the structure of a volume element. A canonical volume element on $M$ is a four-form $\epsilon_{a b c d}$ which is normalized:

$$
g^{a_{1} a_{2}} g^{b_{1} b_{2}} g^{c_{1} c_{2}} g^{d_{1} d_{2}} \epsilon_{a_{1} b_{1} c_{1} d_{1}} \epsilon_{a_{2} b_{2} c_{2} d_{2}}=-4 !
$$

One can then use the volume form to develop a positive definite measure on $M$ (Hawking and Ellis, $1973, \S 2.8$ ). Note that four-forms always exist locally, but not always globally. (This depends only on the structure of the manifold, not the geometry.) So, when they do, the manifold is said to be orientable and a particular four-form is an orientation (structure). However, without (the inverse of) a spacetime metric, there is no way to define the normalization condition to define a unique volume form.

Thus, the spacetime metric provides the structure both for defining "angles" and "volumes". The aspect of that structure pertaining only to the former is called the conformal structure. This is given simply by a continuous assignment of light-cones to the tangent space of the manifold, or in other words, an equivalence class of metrics related to one another by an arbitrary positive smooth scalar field $\Omega$. Given any $g_{a b}, \Omega g_{a b}$ is also a metric of Lorentz signature that assigns exactly the same vectors to be timelike, null, or spacelike at every point, hence also agrees on which curves are timelike, null, and spacelike (Malament, 2012, p. 125).

The spacetime metric (and its inverse) provide the means to determine a different standard of constancy for tensor fields along curves. For simplicity, consider the case of vector fields $X^{a}, Y^{a}$ defined along the image of $\gamma: I \rightarrow M$. One can say that the norm of $X^{a}$, say, is constant along $\gamma$ when $\left\|X^{a}\right\|_{g}$ is so constant, and that the "angle" between $X^{a}$ and $Y^{a}$ is constant along $\gamma$ when $g_{a b} X^{a} Y^{b}$ is so constant. If constancy with respect to an affine connection $\nabla$ implies constancy in this sense, $\nabla$ is said to be compatible with the metric $g_{a b}$. In general, an arbitrarily chosen connection will not be compatible with a given metric, but it general relativity it is required. It turns out that the compatibility of $\nabla$ with $g_{a b}$ is equivalent to the condition that $\nabla_{a} g_{b c}=\mathbf{0}$, i.e., the inner product is preserved by parallel transport (Hawking and Ellis, 1973, p. 40).

Two other pieces of spacetime structure are typically assumed, although they are not definable for all relativistic spacetimes (Hawking and Ellis, 1973, §6.1). The first piece is a spatial orientation, which is a continuous assignment of three ordered linearly independent spacelike vectors to all events in spacetime. The order of the elements determines either a right- or left-hand rule in the usual way; spacetimes that are spatially orientable thus provide the structure to distinguish left and right globally.

The second piece is a temporal orientation. This is a continuous selection of one of the two null cones at every point, the interior of which are designated the future-directed timelike vectors, the others the past-directed ones, and similarly for the null vectors on the cones themselves. These features are inherited by timelike and null curves. A temporal orientation thus allows one to distinguish past from future. It can be determined by any continuous nonvanishing timelike vector field $t^{a}$ on $M$ : in the interior of whichever null cone it falls at a point is the future direction. Consequently, another such field $t^{\prime a}$ yields an equivalent temporal orientation just when $g_{a b} t^{a} t^{\prime b}>0$ everywhere; it yields the opposite orientation just when $g_{a b} t^{a} t^{\prime b}<0$ everywhere instead.

When a spacetime is equipped with a temporal orientation, one can describe its causal structure. This structure consists in two binary relations on the events of $M$. Supposing that $p, q \in M$, then $q$ is said to be in the chronological (resp. causal) future of $p$, written $p \ll q$ (resp. $p \leq q$ ), just 
when there is a continuous, future-directed timelike (resp. causal) curve $\gamma:[a, b] \rightarrow M$ such that $\gamma(a)=p$ and $\gamma(b)=q$. Similarly definitions hold for the chronological (causal) past. The whole causal past of an event $p$ is often interpreted intuitively as the total set of events that could "influence" $p$, and its whole causal future as those that it could "influence"; it is a surprisingly subtle matter to make this idea precise, however (Earman, 2014). Even when a spacetime does not have a temporal orientation, one can still define the two-place relation of chronological (resp. causal) connectibility by dropping the directedness of the above definitions.

\section{What There Is: Alternatives}

The border between alternative formulations of general relativity and alternative relativistic theories of spacetime is vague. Even if one declared the above structures as a definite description of general relativity, it would still be undeniable that many proposed alternatives bear a strong family resemblance to it. Consequently, I have had to draw the line somewhere. My heuristic in doing so had been twofold. First, I consider alternatives that aim to accomplish at least as much as general relativity but with less-or at least different-structure. These are often motivated by conceptual or philosophical considerations that suggest that general relativity posits too much spacetime structure, or structure of the wrong kind. Second, I consider proposals that augment the standard spacetime structure of general relativity by weakening some standard constraint on that structure. These are typically motivated by the desire to represent a wider range of states of affairs using the theory.

Now, the division between and interpretation of these two heuristics is also vague; for example, within the scope of the latter I have not considered additions of extra "gravitational" fields as one finds, for example in Brans-Dicke theory (Brans, 2014). But my hope is that the selection of alternatives presented-for manifold structure in section 3.1 and for geometrical structure in section 3.2-will stimulate more work on the status of alternative structure in general. Regardless of whether any such alternatives should be adopted, such work would strengthen the conceptual and philosophical foundations of general relativity.

\subsection{Alternative Manifold Structure}

The first structure one might question is the collection of events $M$ itself. There is a long history of skeptical arguments towards there being points of space (Forrest, 1996; Coppola and Gerla, 2013), some of which extend to points - events - of spacetime (Russell, 1927, Chs. XXVII-XXX). Many of these are of a metaphysical character; one with a more epistemological focus rests on the claim that one never observes directly the contents of events themselves, but rather spatiotemporally extended processes. Points are then idealized limits of such processes (Geroch, 1978, Ch. 1). However it might be motivated, there have been several approaches to point-free spaces, such as Whitehead's point-free geometry (Whitehead, 1919, 1920; Russell, 1927; Simons, 1987; Gerla and Miranda, 2008), mereotopology (De Laguna, 1922; Whitehead, 1929; Roeper, 1997; Casati and Varzi, 1999; Cohn and Varzi, 2003), and the theory of locales (Johnstone, 1982, 1983), which seek to describe regions by generalizing the algebraic structure of various topological concepts. As far as I am aware, these approaches have not been applied successfully to general relativity, for it is yet unclear how to recover the differentiable structure needed to describe to formulate the necessary 
geometrical structure in the first place (Arntzenius, 2012, Ch. 4). But those sympathetic to this idea might look to alternative methods for describing differentiable structure, such as pseudogroups (Kobayashi and Nomizu, 1963), ringed spaces (Hartshorne, 1977), or sheaves (Tennison, 1975), which depend on being given a topological space, then generalize these methods for point-free spaces—perhaps through the methods of topos theory (Mac Lane and Moerdijk, 1994).

Another alternative approach to events and manifold structure is that of smooth algebras (Nestruev, 2003). Roughly speaking, a smooth algebra is a purely algebraic formulation of the algebra of smooth real functions on a manifold. ${ }^{9}$ Just as fixing a manifold determines its associated algebra of smooth real scalar fields, it turns out that fixing a smooth algebra determines a smooth manifold - with all the trappings of topological and differentiable structure-whose points are reconstructed as homomorphisms of the algebra into $\mathbb{R}$. Indeed, these two mathematical theories are equivalent, in the sense of being categorically dual to one another (Rosenstock et al., 2015).

The motivation for this structural alternative is usually not a philosophical preference for extended regions over points-indeed, there is a sense in which regions are still composed of points in the smooth algebra approach, even though points are themselves not primitive objects there. Rather, by eschewing direct postulation of manifold spacetime itself, it may seem to implement a sort of relationism or empiricism regarding spacetime structure: if the smooth algebra is interpreted as (in some idealized sense) the algebra of world observables, then spacetime events can be seen as merely coincident values of these observables (Earman, 1986, 1989). (I continue the discussion of this alternative in section 3.2, since one can go further, adding the analog of geometrical structure to smooth algebras.)

Another sort of concern comes from the foundations of mathematics, especially over the choice of set-theoretic axioms and the proper set-theoretic commitments of a scientific theory, and from empiricist considerations regarding the observational accessibility of real-valued quantities (i.e., that we can only ever measure quantities with finite precision). There has been some work to see how far one can go letting the local structure of $M$ be only the rationals $\left(\mathbb{Q}^{4}\right)$ and real closed fields (Székely and Madarász, 2013; Székely, 2015), although these must be traded for some sacrifices in representational capacity (as one might expect from forgoing the richness of the real numbers). It remains to be seen whether these sacrifices are from the meat or fat of general relativity.

A different concern with the full structure of the real numbers and sets is that the introduction of numerical coordinates for the spacetime events unduly constrains the kind of structure they can have. Why should spacetime be modeled as a set-theoretic object? Synthetic differential geometry attempts to rectify this by providing an axiomatic rather than constructive (i.e., realanalytic) approach to geometry using the theory of topoi within category theory (Lavendhomme, 1996; Kock, 2006, 2010). In doing so, it provides for a rigorous foundation for infinitesimals within geometry, capturing much of their heuristic use in physics and the engineering sciences, at the expense of a weakening of classical logic to intuitionistic logic. Despite the geometrical tools of the theory being around since the 1980s, and despite its originator Lawvere's original intent to develop the theory as a foundation for continuum mechanics (1980), applications to general relativity have been only preliminary—see Heller and Król (2017) and references therein. Much more work needs to be done to understand what consequences, both conceptual and technical, such a formulation has for spacetime structure.

\footnotetext{
${ }^{9}$ See also Penrose (1968, pp. 141-142) for an approach intermediate between the algebraic approach and the standard one with charts.
} 
Even if one retains the standard topological and differentiable structures of the manifold in terms of sets and real numbers, one might still weaken some of the conditions those structures have been required to satisfy. I consider two of these regarding topological structure, ${ }^{10}$ and two regarding differentiable structure. The first of these concerns the locally Euclidean nature of the manifold, that every point in $M$ has a neighborhood $U$ such that there is homeomorphism $\phi: U \rightarrow$ $\mathbb{R}^{4}$. One can weaken this to add two further types of chart to the differentiable structure: those with boundaries, and with corners. ${ }^{11}$ A chart with boundary (resp. corners) is a homeomorphism $\phi_{1}: U \rightarrow[0, \infty) \times \mathbb{R}^{3}$ (resp. $\phi_{2}: U \rightarrow[0, \infty)^{k} \times \mathbb{R}^{4-k}$ for any $k \leq 4$ ) with $U \subseteq M$. Boundary (resp. corner) points in the manifold are those covered only by charts with boundary (resp. corners).

On the one hand, boundary and corner points are unusual because they are events that are locally unlike typical events: they are the "last" events in one or more spatial or temporal "directions." Any curve passing into such a point in an direction "outward" from the manifold must end there. On the other hand, the singularity theorems of general relativity (for manifolds without boundary) guarantee that under quite generic conditions, a relativistic spacetime will contain incomplete inextendible timelike geodesics, curves that (also) cannot be extended but (from their parameterization) end prematurely. ${ }^{12}$ Although this is a different sense in which the spacetime manifold "ends prematurely," it perhaps undercuts some of the motivation for excluding boundary or corner points in the first place. Furthermore, even setting those issues aside, manifolds with corners can still be used to model collections of events that are strict subcollections of the events of a relativistic universe.

The second topological condition that one could relax is the Hausdorff condition. There are two sorts of contexts where this has been motivated. The first comes from attempts to extend a given spacetime to make it as "large" as it can be-i.e., with as many events as possible. Recall (Hawking and Ellis, 1973, p. 58) that a spacetime $\left(M, g_{a b}\right)$ is extendible when there is another spacetime $\left(M^{\prime}, g_{a b}^{\prime}\right)$ (its extension) and a proper isometric embedding $\psi: M \rightarrow M^{\prime}$; it is inextendible otherwise. It turns out that there exist extendible spacetimes with two classes of null geodesics. In one extension thereof, those in first class are extended and in fact made complete, while the second ones are not and remain incomplete, and vice versa for the second extension. It is not possible for the original spacetime to be extended in both ways at once, unless one relaxes the Hausdorff condition (Hawking and Ellis, 1973, §5.8).

The second context for allowing for non-Hausdorff manifolds comes from a program to represent in the model itself a way in which the future is open, i.e., that there are many distinct continuations of local states of affairs. The most natural implementation of this proposal in general relativity would be to drop the Hausdorff condition, so that at certain collections of events-e.g., along the future light-cones of certain events - the spacetime manifold itself splits into the different alternatives: "such a model is more in accordance with one's intuitive feelings of a determinate past and an indeterminate future than is our normal picture of a Hausdorff space-time" (Penrose, 1979 , p. 594) Although this idea has not found much application in the mainstream literature on

\footnotetext{
${ }^{10}$ One comment on an additional topological assumption, that of second countability: if this is dropped, then there will not exist a globally defined derivative operator. However, the manifold will admit of locally defined derivative operators: could this be enough to formulate a viable analog of general relativity?

${ }^{11}$ Caution: there are many inequivalent definitions of these within the literature. Here I follow Joyce (2012), adapting some inessential details to match the rest of the exposition.

${ }^{12}$ For more discussion of the singularity theorems, see Hawking and Ellis (1973, Chs. 8-10), Wald (1984, Ch. 9.5), and Earman (1995, Ch. 2.8)
} 
general relativity, there has been increasingly sophisticated philosophical work to develop this idea for special (Placek and Belnap, 2012; Müller, 2013) and general relativity (Placek, 2014). There are still a number of challenges (Earman, 2008), but the future is open regarding how this theory of branching spacetimes can be fleshed out.

Turning now to the topological and differentiable structure, there have been some proposals to weaken their compatibility. One way to do so is to keep the differentiable structure but change the topological structure. Hawking et al. (1976) proposed the path topology, the largest topology for which the timelike curves continuous according to the manifold topology are made continuous. ${ }^{13}$ In general, this topology is larger than the manifold topology, but it was constructed explicitly to be more closely connected to and motivated by the geometrical—in particular, causal—structure of spacetime.

Another topology on spacetime events prompted by a similar but still distinct motivation is the Alexandrov (or interval) topology. For any $E \subseteq M$, let $I^{+}(E)=\{q \in M: p \ll q$ for some $p \in E\}$ be the (totality of the) chronological future of $E$, and similarly for the chronological past $I^{-}(E)$. Then the Alexandrov topology on $M$ is the smallest topology in which each $I^{+}(E)$ and $I^{-}(E)$ is open for every $E \subseteq M$. In general the Alexandrov topology is coarser than the manifold topology, but coincides with it just when the spacetime is strongly causal, which is the condition that every neighborhood (in the manifold topology) of $p \in M$ has a sub-neighborhood $U$ such that no continuous causal curve in $M$ intersects $U$ more than once (Hawking and Ellis, 1973, p. 192).

Although both of these proposals provide an alternative topological structure for spacetime determined by some of its causal structure, they can only be cogently interpreted as additional topological structures (giving spacetime the structure of a so-called bitopological space) rather than as replacements. ${ }^{14}$ This is simply because both of them depend on the manifold topology for their definition.

There are other ways to change the differentiable structure while retaining the standard topological structure. Incredibly, for topological manifolds of dimension at least four, it is sometimes possible to find a differentiable structure that is not diffeomorphic to the standard one that is so compatible. Indeed, the exotic version of $\mathbb{R}^{4}$ is homemorphic to the topological product $\mathbb{R} \times \mathbb{R}^{3}$ but not to the corresponding smooth product! These so-called exotic smooth structures are as mathematically surprising as they are difficult to interpret physically for spacetime model. Nevertheless, some tentative conclusions have been drawn (Asselmeyer-Maluga and Brans, 2007). In certain cases the exotic structure can be localized to a region of the manifold, acting as a "source" for gravitation in the sense of admitting only of a connection that is non-flat in that region (Brans, 1994; Sładkowski, 2001; Asselmeyer-Maluga and Brans, 2015). And because the manifold of a globally hyperbolic spacetime can always be smoothly decomposed into $\mathbb{R} \times \Sigma$ for some smooth three-manifold $\Sigma$, it has recently been argued that spacetimes with exotic smooth structures for $\mathbb{R}^{4}$ provide a counterexample to the strong cosmic censorship conjecture (Etesi, 2015), roughly that generic spacetimes satisfying some relevant energy condition are globally hyperbolic (but cf. Earman (1995, pp. 45-46)). Much more work needs to be done to understand if there is an acceptable interpretation of these exotica and their bearing on other aspects of spacetime structure, such as the presence of singularities (Earman, 1995, p. 50).

\footnotetext{
${ }^{13}$ Strictly speaking, they restricted their definition to strongly causal spacetimes, defined presently. For a review of related proposals, see Saraykar and Janardhan (2016).

${ }^{14}$ See Heathcote (1988) for a discussion of the interpretive relevance of the path topology in particular.
} 


\subsection{Alternative Geometrical Structure}

One way to introduce additional geometrical structure is by relaxing some of the constraints on the affine connection. For example, if the requirement that it be torsion-free is dropped, as Cartan $(1922,1923 a, b, 1924)$ investigated in a series of papers, ${ }^{15}$ the result, now known as EinsteinCartan theory, allows for distinct dynamics for particles with intrinsic angular momentum (Hehl et al., 1976; De Sabbata and Sivaram, 1994; Trautman, 2006). ${ }^{16}$ The torsion tensor is then related to a spin tensor representing the intrinsic angular momentum density of matter fields (and their interactions) in spacetime. ${ }^{17}$ One can further drop the requirement that the connection be compatible with the metric (Hehl et al., 1995); the degree to which this occurs is represented by the nonmetricity tensor $\nabla_{a} g_{b c}$. The dynamical equations for this "metric-affine" theory are then specified by some Lagrangian function of the scalar curvature (as is the case in standard general relativity) and these new geometrical structures. Both the Einstein-Cartan and metric-affine theories have been suggested as better accounts of the "microstructure" of the dynamics of spacetime with matter (Stachel, 1999), in particular the symmetries found in theories of particle physics, but there is still some controversy regarding whether they are ultimately equivalent to theories with more complicated matter fields but with only the usual spacetime structure. ${ }^{18}$

Relaxations of the smoothness constraint on the metric tensor, although they don't introduce new geometrical structures per se, are worth mentioning. First, the metric need only be twice differentiable to define the Einstein tensor (for which see section 4). Alternately, if it is only continuous but with locally square integrable weak first derivatives, then the equation is defined in a distributional sense (Hawking and Ellis, 1973, pp. 57-58). ${ }^{19}$ When complemented with distributional matter sources of some (but not all) types, this allows one to model shock waves, thin shells of matter, cosmic strings, etc. (Geroch and Traschen, 1987), or to provide spacetime extensions unavailable when smoothness is demanded (Galloway and Ling, 2017); when the first derivatives of the metric are not Lipschitz continuous in particular, they allow for indeterministic geodesic trajectories (Fletcher, 2017).

There have been some proposals to try to reduce the geometrical structure that standard general relativity posits without losing any substantive possibilities or properties that the theory can represent. One that picks up its point of development from the smooth algebras discussed in section 3.1 are Einstein algebras (Geroch, 1972): they augment smooth algebras with further structure that amounts to the standard geometrical structure of the spacetime metric. Earman $(1986,1989)$ had suggested this approach to provide a spacetime structure that blocks a formulation of the hole

\footnotetext{
${ }^{15}$ For English translations of Cartan (1922) and Cartan (1923a,b, 1924), respectively, see Cartan (1980) and Cartan (1986).

${ }^{16}$ Einstein-Cartan theory is thus in a sense also a generalization of teleparallel gravity (Aldrovandi and Pereira, 2012), in which one allows for torsion but assumes that the curvature vanishes, although this does not mean that these objects play the same role in that theory (Knox, 2011) or in Einstein-Cartan theory (Knox, 2013). More could be done to present these theories within a comprehensible unified conceptual framework.

${ }^{17}$ A different sort of additional structure used to model particles with intrinsic angular momentum is spinor structure, which is definable on an orientable manifold just in case it is parallelizable, i.e., has a continuous section of its (tangent) frame bundle (Geroch, 1968). Although spinor structure provides an elegant treatment of various topics, it is representable from tensor fields (Penrose and Rindler, 1984).

${ }^{18}$ In the case of Einstein-Cartan theory, the equivalence may depend on the type of spin coupling (Trautman, 2006). For further extensive replies to objections, see Hehl and Obukhov (2007).

${ }^{19}$ An intermediate position that avoids the use of distributions holds that the metric is everywhere $C^{1}$ but piecewise $C^{3}$ (Schild, 1967, §7).
} 
argument (Norton, 2015). It turns out that Einstein algebras are in a precise sense equivalent with Lorentzian geometries (Rosenstock et al., 2015), so it is less clear both that it avoids an analogous formulation of the hole argument and that the alternative structure it posits is really just different in appearance, not in substance (Rynasiewicz, 1992). ${ }^{20}$

A idea different from Earman's but towards the same goal is to take all the models of the standard spacetime structure and then form equivalence classes under the orbits of the isometry group (Iftime and Stachel, 2006). In practice, though, it seems one must almost always work with a representative from the class, and having many isometric models with the same representational capacities can be useful. Given that there are resolutions of the hole argument that do not require this awkward move (Weatherall, 2018; Pooley, 2013, §7), it is not clear how well motivated it is in the end.

Another type of quotient one might apply to reduce the geometrical structure is that of the homothety group, which, for a given spacetime metric $g_{a b}$ is just the one-parameter group of transformations given by $g_{a b} \mapsto \lambda g_{a b}$ for $\lambda>0$. Although this is not motivated by the hole argument, it does capture the former proposal's underlying idea that perhaps there is surplus representational structure in general relativity, in this case with its representation of the units of spatiotemporal quantities. If one recalls from section 2.2 that the spacetime metric determines both durations and distances, then homothetic transformations might plausibly be interpreted as scale transformations for these quantities. Indeed, homothetically related spacetimes are often glossed as differing only by a choice of units. If this is correct, it would be an attractive elimination of structure for comparativists about quantities (Dasgupta, 2013; Eddon, 2013), but more work needs to be done to assess its cogency. ${ }^{21}$

\section{What Determines What: The Standard Account}

The standard presentation of a relativistic spacetime achieves a remarkable reduction of structure: very much is determined by relativity little. In particular, one can do with just the following: $\left(M, \mathcal{A}, g_{a b}\right)$. Here, $M$ is the collection of events and $\mathcal{A}$ is an atlas for $M$ with some particular properties, which determines all other manifold structures. For geometrical structure, the metric $g_{a b}$ determines all else. If the manifold is orientable, then one needs to add only a temporal orientation $t^{a}$ to the geometrical structure.

\subsection{Standard Determination of Manifold Structure}

Both the differentiable and topological structure of the manifold can be determined by an appropriate atlas. In particular, if the atlas satisfies certain analogs of the conditions on the topological structure discussed in section 2.1, then the topological structure determined will also have those features (Malament, 2012, p. 4).

\footnotetext{
${ }^{20}$ Other presumably equivalent reformulations of standard general relativity (perhaps modulo some technical conditions) include those in terms of gauge theory (Blagojević and Hehl, 2013) — that is, using the mathematics of principal bundles - and geometric algebra (Lasenby et al., 1998). Within the former, the Cartan formalism emphasizes the use of frame fields and the exterior calculus.

${ }^{21}$ When, say, matter theories formulated in a general relativistic spacetime introduce different spatiotemporal scales, it is no longer clear whether homotheties could still be interpreted as scale transformations. For such matter-augmented models, then, quotienting by the homothety group may identify models that are not representationally equivalent.
} 
So, take $\mathcal{A}$ to be a maximal, smooth $\left(C^{\infty}\right.$ differentiable) structure on $M$ satisfying the following conditions:

1. It is Hausdorff, i.e., for any distinct $p, p^{\prime} \in M$, there are charts $\phi, \phi^{\prime} \in \mathcal{A}$ with domains $U, U^{\prime} \subseteq M$ such that $p \in U, p^{\prime} \in U^{\prime}$, and $U \cap U^{\prime}=\emptyset$.

2. It is path-connected, in the sense that for every $p, q \in M$, there is a function $f:[0,1] \rightarrow M$ such that $f(0)=p, f(1)=q$, and whenever $f(x) \in U$ for some chart $\phi: U \rightarrow \mathbb{R}^{4}$ in $\mathcal{A}$, then $(\phi \circ f)_{\mid f^{-1}[f[0,1] \cap U]}$ is a smooth function.

3. It contains a countable differentiable substructure, i.e., there is a countable differentiable structure $\mathcal{A}^{\prime} \subset \mathcal{A} .^{22}$

This is the atlas used in the standard presentation of a relativistic spacetime. Instead of requiring compatibility between this structure and a separately defined topology on $M$, the topological structure on $M$ is rather determined as that coherent with the domains of the charts of $\mathcal{A}$, i.e., the smallest topology on $M$ for which all the charts are continuous. The resulting topological structure then makes $M$ a Hausdorff, connected, second-countable topological four-manifold (O'Neill, 1983, p. 23).

Two further features regarding determination of manifold structure are of note. First, any $C^{k}$ differentiable structure $\mathcal{A}$ on a set $M$ for $k \geq 1$ determines a unique, maximal $C^{\infty}$ differentiable structure on $M$, in the sense that there is a unique such structure whose charts are compatible with those of $\mathcal{A}$ (Whitney, 1936). This is pragmatically important, for it permits one great economy in expressing a spacetime model: one need only describe some $C^{k}$ differentiable structure or other, which can often be done with only a few charts. But this determination is not typically incorporated as a further reduction of manifold structure, for on its face it would tend to suggest misleadingly that certain charts are somehow representationally privileged based on the manifold structure alone. ${ }^{23}$ Second, there the global existence of a derivative operator implies (and is implied by) the countable differentiable substructure condition (Geroch, 1971). In fact, this condition is implied by the existence of a spacetime metric. So, one could leave that condition off of the list above as a redundancy.

\subsection{Standard Determination of Geometrical Structure}

On the standard account, the spacetime metric $g_{a b}$ determines all the other geometrical structure described in section 2.2 (except for a temporal and spatial orientations, if they exist). I have already described how it determines the inner product (null cone) and norm structures on the tangent space at each point-hence, the conformal structure and the relation of causal connectability as well. Once a smooth Lorentz-signature metric is specified, it also determines a unique globally defined torsion-free derivative operator, the Levi-Civita derivative operator, which can be explicitly constructed from the metric (and an arbitrarily chosen derivative operator). A unique derivative

\footnotetext{
${ }^{22}$ This is the analog of the Lindelöf condition-see footnote 7.

${ }^{23}$ The issue is that it is not transparent what the category of (not necessarily maximal) differentiable structures is supposed to be, i.e., what their relevant notion of isomorphism is. One might think that it is the same as maximal differentiable structures_namely, diffeomorphisms - so that two differentiable structure with a common maximal extension would be isomorphic, but then it becomes unclear just how they determine further structure.
} 
operator in turn determines a unique curvature tensor and a unique projective structure - the class of all pregeodesics on the spacetime. In fact, it determines the geodesics as well: these are just the pregeodesics whose tangent vectors satisfy the geodesic equation with respect to the Levi-Civita derivative operator.

The only geometrical structures not quite determined by the spacetime metric concern orientability of various sorts. It turns out, though, that any two of the following three conditions imply the third (Hawking and Ellis, 1973, §6.1):

1. Spacetime is orientable: there exists a global four-form on $M$.

2. Spacetime is temporally orientable: there exists a continuous timelike vector field on $M$.

3. Spacetime is spatially orientable: there exists a continuous spatial frame field on $M$.

So, when the spacetime is orientable, the metric determines the volume element (hence the volumetric structure of spacetime) according to its normalization condition. In this case, the addition of a temporal (resp. spatial) orientation as geometrical structure on $M$ determines a spatial (resp. temporal) orientation on $M$. It is most typical to assume a temporal orientation, so that causal structure is then determined (as the orientation allows one to split the causal connectability relation into two, based on past and future).

Readers with enough familiarity with the general theory of relativity may notice that I have not yet mentioned anything about matter fields on spacetime, or about Einstein's field equation, which connects their energetic properties with spacetime curvature. This is because, on the standard account, matter fields and their energetic properties are not a part of spacetime structure, even though there is a sense in which they are all intimately connected. To show this requires some preliminary definitions.

The Ricci tensor $R_{a b}$ associated with an affine connection is defined as the contraction of the associated curvature tensor: $R_{a b}=R_{a b c}^{c}{ }^{24}$ The scalar curvature field $R$, meanwhile, is defined from the Ricci tensor in the presence of a metric: $R=g^{b a} R_{a b}$. These two fields are combined into the Einstein tensor $G_{a b}=R_{a b}-\frac{1}{2} R g_{a b}$. Einstein's field equation then states (in units where Newton's constant and the speed of light have have been set to one) that $G_{a b}=8 \pi T_{a b}$, where $T_{a b}$ is the energy-momentum tensor associated with all matter fields and their interactions. ${ }^{25}$ It encodes the energy densities and three-momentum densities that would be measured relative to all observers at each event of spacetime (Malament, 2012, Ch. 2.5).

We see from Einstein's field equation that the energy-momentum tensor is in fact determined by the Einstein tensor, which is in turn determined by the spacetime metric, for the Ricci tensor is just that associated with the Levi-Civita connection. However, on the standard account this does not imply that the metric determines matter. For the matter fields on spacetime also determine the energy-momentum tensor, but not vice versa: distinct matter fields on a spacetime can generate the same energy-momentum tensors. From one perspective, it is just this overdetermination which makes general relativity a theory from which it is difficult to extract exact solutions, i.e., jointly consistent descriptions of manifold, metric, and matter according to the aforementioned structural constraints.

\footnotetext{
${ }^{24}$ Caution: not all references use the same index placement convention for the definition of the Ricci tensor, and similarly for the sign convention for the metric, the Einstein tensor (for which see below), etc. (Misner et al., 1973).

${ }^{25}$ I have omitted the term $\Lambda g_{a b}$, where $\Lambda$ is the cosmological constant. Including it might change whether the energy-momentum tensor is determined by the metric, depending on its interpretation (Earman, 2003).
} 


\section{What Determines What: Alternatives}

Before turning to various alternatives concerning what determines what, it will be helpful to review the converses of the standard determination relations outlined in section 4. As the existence of exotic smooth structure (discussed in section 3.1) attests, in general the topological structure does not determine the differentiable structure. ${ }^{26}$ An affine connection $\nabla$, even if it is torsion-free, does not determine a Lorentz metric in at least two ways. First, there may not be any metric $g_{a b}$ such that $\nabla_{c} g_{a b}=\mathbf{0} .{ }^{27}$ Second, even if there is such a metric, its signature is not in general determined; even if that is provided the metric is not in general determined, even up to homothety (Hall, 2004, Theorem 9.2). Similarly, the curvature structure does not determine the affine connection either. ${ }^{28}$

What about the conformal and projective structure? Naturally, the conformal structure only determines the metric up to a conformal factor-that is, the class $\Omega^{2} g_{a b}$ for positive smooth scalar fields $\Omega .{ }^{29}$ Interestingly, though, the conformal structure does determine which null curves are pregeodesics - and vice versa-for it is only this class of pregeodesics which is invariant under arbitrary conformal transformations (Malament, 2012, p. 125). (This will play an important role in the discussion of the approach by Ehlers et al. (1972) discussed below.) Similarly, the projective structure does not determine the affine connection. ${ }^{30}$ But, a famous theorem of Weyl (1921) shows that two spacetimes with the same conformal and projective structures are in fact homothetically related.

It is tempting to conclude that Weyl's theorem shows that conformal and projective structure determine metric structure up to a homothety, but this is not quite correct: one must assume in the first place that the conformal and projective structures already are determined by a metric. However, in a remarkable paper, Ehlers et al. (1972) investigated the circumstances under which one could make such a determination. In fact, they go much further than this, providing an informal axiomatic structure characterizing the aspects of this determination.

Here is a sketch of their framework: Their first class of axioms establishes the manifold structure by supposing that each particle worldline has the structure of a one-dimensional manifold and that all events can be covered by radar coordinate systems. The second concerns the propagation of light, which establishes the conformal structure by assuming that the directions of light propagation bound those of particles into two connected components - what end up being the null cones. The third concerns the worldlines of free particles, which are assumed to have a coordinate representation in terms of straight lines, thereby determining a projective structure up to a choice of

\footnotetext{
${ }^{26}$ No general statement is yet known since the analysis of exotica in four dimensions has proceeded on a case-bycase basis, with major work so far focusing on non-compact four-manifolds.

${ }^{27}$ It is necessary and sufficient that the connection's holonomy group be a subgroup of the orthogonal group corresponding to the metric signature (Schmidt, 1973) — see also Atkins (2008) for a different characterization. There are pointwise conditions any such connection must satisfy if it is to be a metric connection locally, but a connection being locally metric everywhere does not make it globally so.

${ }^{28}$ Here the question is complicated somewhat by how one decides to treat torsion, and whether one wishes to determine the connection only or a metric connection in particular (which, in the mathematics literature, is known as the problem of prescribed curvature). See Hall (2004, Ch. 9.3) for a treatment of the problem assuming that the curvature structure is associated already with the Levi-Civita connection of some Lorentz metric.

${ }^{29}$ Such conformally equivalent metrics also have the same Weyl conformal tensor, but the converse does not hold unless they are conformally flat, i.e., unless the Weyl tensor vanishes (Malament, 2012, p. 85).

${ }^{30}$ Projectively equivalent connections have the same Weyl projective tensor, but the converse does not hold (Hall, 2013).
} 
torsion, assumed to vanish, according to which the worldlines of the free particles are geodesics. The fourth class of axioms postulates the compatibility of the conformal and projective structures, entailing that the worldlines of light rays are also geodesics of the projective structure. This compatibility then determines a Lorentz metric up to a conformal transformation. The resulting space, called a Weyl geometry, ${ }^{31}$ only yields a relativistic spacetime up to homothety when one further condition is imposed: that there is no second clock effect. This is the statement, in accordance with experimental evidence, that the rates of ideal clocks (i.e., the scale factor of their affine parameter) is not path-dependent: two ideal clocks at an initial event whose rates are in sync will still be in sync at any common other event. Because of the multifaceted richness of their approach, many alternatives have been proposed that improve upon various aspects of their the construction, such as the representation of projective structure, its compatibility with the conformal structure, and even the use of particles as primitive entities. ${ }^{32}$

These works all have a certain operational subtext that prioritizes material constituents over spatiotemporal structure. The most famous in this empiricist tradition emanates from Mach, whose appellated principle states that in some sense, the matter content of the universe determines its spacetime structure, in particular its inertial structure (which in general relativity is implemented by the projective structure or the affine connection or some related structure). ${ }^{33}$ It is still not yet clear how some determination of this sort could be made: specifying the energy-momentum $T_{a b}$ in Einstein's field equation does not even determine the full Riemann curvature tensor, much less the affine connection or metric; moreover, for most matter fields, their associated energy-momentum tensors cannot even be stated without invoking the metric (Malament, 2012, pp. 159-160). Indeed, although Einstein was motivated by some version of Mach's principle in his development of the general theory of relativity, in the end he agreed it was not so implemented (Torretti, 1996, Ch. 6.2). Nevertheless, there is some indication that a version of the principle might hold in a restricted class of spacetime, in particular those with certain types of boundary conditions. ${ }^{34}$

A different approach in a similar spirit aims to show that the metric can be determined not from the distribution of energy-momentum of matter, but from the detailed dynamical equations of the matter fields themselves. Hehl and collaborators (Itin and Hehl, 2004; Hehl and Obukhov, 2005, 2006) have proposed a determination of the spacetime metric through an appropriate "premetric" version of electromagnetism. Naturally, this theory requires further assumptions and constraints which well deserve further attention. Perhaps as well the approach could be generalized to include any matter theory formalized in terms of quasilinear hyperbolic systems of equations, which surprisingly seems to include almost all classical fields of interest (Geroch, 2011).

A third approach is the "dynamical relativity" program of Brown (2006) influential among of philosophers of physics, which seeks to show how the metric field encodes durations and lengths not by fiat, but through its coupling as a physical field to more ponderable matter fields. Al-

\footnotetext{
${ }^{31}$ The reference is to Weyl's early theory, of which this geometry is a model. For historical analysis, see Ryckman (2004).

${ }^{32}$ For commentary and references, see Ehlers (1973), Sklar (1977), Trautman (2012), and Pfister and King (2015).

${ }^{33}$ There are in fact many different version of this informal idea. For a variety of perspectives, see Barbour and Pfister (1995).

${ }^{34}$ The shape dynamics program of Barbour and collaborators is also a Machian program which aims to describe relativistic spacetimes as collection of scale-invariant Riemannian three-geometries; thus, it only seeks to recover globally hyperbolic spacetimes with certain boundary conditions. See Pooley $(2013, \S 6.2)$ for an accessible review and references.
} 
though there continues to be controversy about how to interpret the program, one version, due to Knox (2013, forthcoming), takes it to claim that symmetry properties of the equations of motion for matter fields determine spacetime structure, which should be understood functionally as whatever provides inertial structure. Inertial structure, in turn, is understood through inertial coordinates charts generated from frame fields on timelike geodesics. This is supposed to succeed as a determination of spacetime structure because Ehlers et al. (1972) proved that "the full set of timelike geodesics (the inertial trajectories) ... is sufficient to fix both conformal and projective structure. This in turn fixes metric structure up to a global scale factor" (Knox, 2013, p. 349), i.e., a homothety. However, even the cursory review of Ehlers et al. (1972) above shows that no such determination has been proven unless a number of substantive non-inertial assumptions are made. Projective structure itself (i.e., the inertial trajectories) does not determine conformal structure, and even given a Lorentzian metric and its Levi-Civita connection on a four-manifold, that metric is not the unique Lorentz metric compatible with that connection, even up to homothety. There is a subtlety in this last negative conclusion, however: the failure of uniqueness up to homothety depends on the spacetime having nontrivial symmetries. ${ }^{35}$ Symmetric spacetimes are not a new problem for the dynamical approach (Norton, 2008; Pooley, 2013, pp. 573-574), but these considerations show that Knox's particular brand of functionalism about spacetime structure does not avoid it.

In addition to these approaches that seek to determine other structure from that of matter, there is another venerable tradition in which causal structure takes the role of matter. Robb $(1914,1921$, 1936) showed how the metric of Minkowski spacetime can be determined up to homothety from a single relation of causal precedence on an uncountable domain, and some results are known regarding how this could be weakened to a countable domain (Sen, 2010, Part I). Zeeman (1964) revealed accordingly that the causal isomorphisms of Minkowski spacetime $\left(M, \eta_{a b}\right)$-i.e., the bijections such that for all $p, q \in M, p \ll q$ if and only if $\phi(p) \ll \phi(q)$-are exactly the homotheties composed with the Poincaré tranformations, i.e., the maximal homothety group for any spacetime (Hall, 2004, p. 293).

It is important to note that these results do not extend verbatim to general relativity, where one cannot hope that conformal structure alone determines the metric. But with some additional constraints, a version of Zeeman's theorem does. In order to describe it, one more definition is needed. A spacetime $\left(M, g_{a b}\right)$ with a temporal orientation is future- (resp. past-)distinguishing when, for all $p, q \in M, I^{+}(p)=I^{+}(q)$ (resp. $\left.I^{-}(p)=I^{-}(q)\right)$ implies that $p=q$; it is distinguishing when it is both future- and past-distinguishing. Malament (1977) then showed that given any two distinguishing, temporally oriented spacetimes $\left(M, g_{a b}\right)$ and $\left(M^{\prime}, g_{a b}^{\prime}\right)$, if $\phi: M \rightarrow M^{\prime}$ is a causal isomorphism, then $\phi$ is in fact a conformal isometry. This result might be glossed informally as "causality implies the conformal group" or "causal structure determines conformal structure," but like with Weyl's theorem, one must take some care: the determination only holds for causal structures themselves determined from distinguishing spacetimes.

\footnotetext{
${ }^{35}$ When the manifold is simply connected, the set of metrics on that manifold for which the desired functional determination is valid is in fact generic in the $C^{\infty}$ open (or Whitney) topology on those metrics (Hall, 2004, pp. 258259). But it is not yet clear whether this is the relevant topology with which to evaluate this question (Fletcher, 2016): does this verdict change if one uses a less problematic topology capturing global similarity (Fletcher, 2018)?
} 


\section{References}

Aldrovandi, R. and Pereira, J. G. (2012), Teleparallel Gravity: An Introduction, Springer, Dordrecht.

Arntzenius, F. (2012), Space, Time and Stuff, Oxford University Press, Oxford.

Asselmeyer-Maluga, T. and Brans, C. (2015), 'How to include fermions into general relativity by exotic smoothness', General Relativity and Gravitation 47, 30.

Asselmeyer-Maluga, T. and Brans, C. H. (2007), Exotic Smoothness and Physics: Differential Topology and Spacetime Models, World Scientific, Singapore.

Atkins, R. (2008), 'When is a connection a metric connection?', New Zealand Journal of Mathematics 38, 225-238.

Barbour, J. B. and Pfister, H., eds (1995), Mach's Principle: From Newton's Bucket to Quantum Gravity, Vol. 6 of Einstein Studies, Birkhäuser, Boston.

Barrett, T. W. (2015), 'Spacetime structure', Studies in History and Philosophy of Modern Physics 51, 37-43.

Beem, J. K., Ehrlich, P. and Easley, K. (1996), Global Lorentzian Geometry, 2nd edn, CRC Press, Boca Raton.

Blagojević, M. and Hehl, F. W. (2013), Gauge Theories of Gravitation: A Reader with Commentaries, World Scientific, Singapore.

Brans, C. (1994), 'Localized exotic smoothness', Classical and Quantum Gravity 11, 1785-1792.

Brans, C. H. (2014), 'Jordan-Brans-Dicke Theory’, Scholarpedia 9(4), 31358. revision \#151619.

Brown, H. R. (2006), Physical Relativity: Space-time structure from a dynamical perspective, Oxford University Press, Oxford.

Carnap, R. (1922), Der Raum: Ein Beitrag zur Wissenschaftslehre, Reuther \& Reichard, Berlin. (Kant-Studien Ergänzungshefte, no.56).

Cartan, É. (1922), 'Sur une généralisation de la notion de courbure de Riemann et les espaces à torsion', Comptes rendus de l’Académie des sciences 174, 593-595.

Cartan, É. (1923a), 'Sur les variétés à connexion affine et la théorie de la relativité généralisée, Part I', Annales Scientifiques de l'École Normale Supérieure 40, 325-412.

Cartan, É. (1923b), 'Sur les variétés à connexion affine et la théorie de la relativité généralisée, Part I', Annales Scientifiques de l'École Normale Supérieure 41, 1-25.

Cartan, É. (1924), 'Sur les variétés à connexion affine et la théorie de la relativité généralisée, Part II', Annales Scientifiques de l'École Normale Supérieure 42, 17-88. 
Cartan, É. (1980), On a generalization of the notion of Riemann curvature and spaces with torsion, in P. G. Bergmann and V. De Sabbata, eds, 'Cosmology and Gravitation: Spin, Torsion, Rotation, and Supergravity’, Plenum, New York, pp. 489-492. Trans. G. D. Kerlick.

Cartan, É. (1986), On Manifolds with an Affine Connection and the Theory of General Relativity, Bibliopolis, Naples. Trans. A. Magnon and A. Ashtekar.

Casati, R. and Varzi, A. (1999), Parts and places: the structures of spatial representation, MIT Press, Cambridge, MA.

Cohn, A. G. and Varzi, A. C. (2003), 'Mereotopological connection', Journal of Philosophical Logic 32, 357-390.

Coppola, C. and Gerla, G. (2013), 'Special issue on point-free geometry and topology', Logic and Logical Philosophy 22(2), 139-143.

Dasgupta, S. (2013), Absolutism vs comparativism about quantity, in K. Bennett and D. W. Zimmerman, eds, 'Oxford Studies in Metaphysics', Vol. 8, Oxford University Press, Oxford, pp. 105-148.

De Laguna, T. (1922), 'Point, line and surface as sets of solids', The Journal of Philosophy 19, 449_ 461.

De Sabbata, V. and Sivaram, C. (1994), Spin and Torsion in Gravitation, World Scientific, Singapore.

Earman, J. (1986), 'Why space is not a substance (at least not to first degree)', Pacific Philosophical Quarterly 67(4), 225-244.

Earman, J. (1989), World Enough and Space-Time: Absolute versus Relational Theories of Space and Time, MIT Press, Cambridge, MA.

Earman, J. (1995), Bangs, Crunches, Wimpers, and Shrieks: Singularities and Acausalities in Relativistic Spacetimes, Oxford University Press, Oxford.

Earman, J. (2003), 'The cosmological constant, the fate of the universe, unimodular gravity, and all that', Studies in History and Philosophy of Modern Physics 34(4), 559-577.

Earman, J. (2008), Pruning some branches from "branching spacetimes", in D. Dieks, ed., 'The Ontology of Spacetime II', Elsevier, Amsterdam, pp. 187-205.

Earman, J. (2014), 'No superluminal propagation for classical relativistic and relativistic quantum fields', Studies in History and Philosophy of Modern Physics 48, 102-108.

Eddon, M. (2013), 'Quantitative properties', Philosophy Compass 8(7), 633-645.

Ehlers, J. (1973), The nature and structure of spacetime, in J. Mehra, ed., 'The Physicist's Conception of Nature', Kluwer, Dordrecht, pp. 71-91. 
Ehlers, J., Pirani, F. A. E. and Schild, A. (1972), The geometry of free fall and light propagation, in L. O'Reifeartaigh, ed., 'General Relativity, papers in honour of J. L. Synge', Clarendon Press, New York, pp. 63-84. Republished as Ehlers et al. (2012).

Ehlers, J., Pirani, F. A. E. and Schild, A. (2012), 'Republication of: The geometry of free fall and light propagation', General Relativity and Gravitation 44(6), 1587-1609.

Etesi, G. (2015), 'Exotica or the failure of the strong cosmic censorship in four dimensions', International Journal of Geometric Methods in Modern Physics 12, 1550121-1-1550121-14.

Fletcher, S. C. (2016), 'Similarity, topology, and physical significance in relativity theory', British Journal for the Philosophy of Science 67(2), 365-389.

Fletcher, S. C. (2017), Indeterminism, gravitation, and spacetime theory, in G. Hofer-Szabó and L. Wroński, eds, 'Making it Formally Explicit: Probability, Causality and Indeterminism', Springer, Cham, pp. 179-191.

Fletcher, S. C. (2018), 'Global spacetime similarity', Journal of Mathematical Physics 59(11), 112501.

Forrest, P. (1996), 'From ontology to topology in the theory of regions', The Monist 79, 34-50.

Galloway, G. J. and Ling, E. (2017), 'Some remarks on the $C^{0}$-(in)extendibility of spacetimes', Annales Henri Poincaré 18(10), 3427-3447.

Gerla, G. and Miranda, A. (2008), Mathematical features of Whitehead's point-free geometry, in M. Weber and J. Desmond, William, eds, 'Handbook of Whiteheadian Process Thought', Vol. 2, Ontos Verlag, Frankfurt, pp. 121-132.

Geroch, R. (1971), Spacetime structure from a global viewpoint, in B. K. Sachs, ed., 'General Relativity and Cosmology', Academic Press, New York, pp. 71-103.

Geroch, R. (1978), General Relativity from A to B, University of Chicago Press, Chicago.

Geroch, R. (2011), Faster than light?, in M. Plaue, A. D. Rendall and M. Scherfner, eds, 'Advances in Lorentzian Geometry: Proceedings of the Lorentzian Geometry Conference in Berlin', American Mathematical Society, Providence, pp. 59-70.

Geroch, R. and Horowitz, G. T. (1979), Global structure of spacetimes, in S. W. Hawking and W. Israel, eds, 'General Relativity: An Einstein Centenary Survey', Cambridge University Press, Cambridge, pp. 212-293.

Geroch, R. P. (1968), 'Spinor structure of space-times in general relativity, I', Journal of Mathematical Physics 9, 1739-1744.

Geroch, R. P. (1972), 'Einstein algebras', Communications in Mathematical Physics 26, 271-275.

Geroch, R. and Traschen, J. (1987), 'Strings and other distributional sources in general relativity', Phys. Rev. D 36, 1017-1031. 
Hall, G. (2013), 'On the converse of Weyl's conformal and projective theorems', Publications de l'Institut Mathématique 94(108), 55-65.

Hall, G. S. (2004), Symmetries and Curvature Structure in General Relativity, World Scientific, Singapore.

Hartshorne, R. (1977), Algebraic Geometry, Springer, New York.

Hawking, S. W. and Ellis, G. F. R. (1973), The large scale structure of space-time, Cambridge University Press, Cambridge.

Hawking, S. W., King, A. R. and McCarthy, P. J. (1976), 'A new topology for curved space-time which incorporates the causal, differential, and conformal structures', Journal of Mathematical Physics 17(2), 174-181.

Heathcote, A. (1988), 'Zeeman-Göbel topologies', British Journal for the Philosophy of Science 39(2), 247-261.

Hehl, F. and Obukhov, Y. (2006), Spacetime metric from local and linear electrodynamics: A new axiomatic scheme, in J. Ehlers and C. Lämmerzahl, eds, 'Special Relativity: Will it Survive the Next 101 Years?', Springer, Berlin, pp. 163-187.

Hehl, F. W., McCrea, J. D., Mielke, E. W. and Ne'eman, Y. (1995), 'Metric-affine gauge theory of gravity: field equations, Noether identities, world spinors, and breaking of dilation invariance', Physics Reports 258, 1-171.

Hehl, F. W. and Obukhov, Y. N. (2005), 'To consider the electromagnetic field as fundamental, and the metric only as a subsidiary field', Foundations of Physics 35(12), 2007-2025.

Hehl, F. W. and Obukhov, Y. N. (2007), 'Élie Cartan's torsion in geometry and in field theory, an essay', Annales de la Fondation Louis de Broglie 32(2-3), 157-194.

Hehl, F. W., von der Heyde, P., Kerlick, G. D. and Nester, J. M. (1976), 'General relativity with spin and torsion: Foundations and prospects', Reviews of Modern Physics 48, 393-416.

Heller, M. and Król, J. (2017), ‘Infinitesimal structure of singularities’, Universe 3, 16.

Iftime, M. and Stachel, J. (2006), 'The hole argument for covariant theories', General Relativity and Gravitation 38, 1241-1252.

Itin, Y. and Hehl, F. W. (2004), 'Is the Lorentz signature of the metric of spacetime electromagnetic in origin?', Annals of Physics 312(1), 60-83.

Johnstone, P. (1982), Stone Spaces, Cambridge University Press, Cambridge.

Johnstone, P. (1983), 'The point of pointless topology', Bulletin of the American Mathematical Society (New Series) 8(1), 41-53.

Joyce, D. (2012), On manifolds with corners, in S. Janeczko, J. Li and D. H. Phong, eds, 'Advances in Geometric Analysis', International Press, Boston, pp. 225-258. 
Knox, E. (2011), 'Newton-Cartan theory and teleparallel gravity: The force of a formulation', Studies in History and Philosophy of Modern Physics 42(4), 264 - 275.

Knox, E. (2013), 'Effective spacetime geometry', Studies in History and Philosophy of Modern Physics 44, 346-356.

Knox, E. (forthcoming), 'Physical relativity from a functionalist perspective', Studies in History and Philosophy of Modern Physics .

Kobayashi, S. and Nomizu, K. (1963), Foundations of Differential Geometry, Vol. 1, Interscience, New York.

Kobayashi, S. and Nomizu, K. (1969), Foundations of Differential Geometry, Vol. 2, Interscience, New York.

Kock, A. (2006), Synthetic Differential Geometry, 2nd edn, Cambridge University Press, Cambridge.

Kock, A. (2010), Synthetic Geometry of Manifolds, Cambridge University Press, Cambridge.

Lasenby, A., Doran, C. and Gull, S. (1998), 'Gravity, gauge theories and geometric algebra', Philosophical Transactions of the Royal Society A 356, 487-582.

Lavendhomme, R. (1996), Basic Concepts of Synthetic Differential Geometry, Kluwer, Dordrecht.

Lawvere, F. W. (1980), 'Toward the description in a smooth topos of the dynamically possible motions and deformations of a continuous body', Cahiers de Topologie et Géométrie Différentielle Catégoriques 21(4), 377-392.

Lee, J. M. (2011), Introduction to Topological Manifolds, 2nd edn, Springer, New York.

Lewis, D. K. (1986), On the Plurality of Worlds, Blackwell, Oxford.

Mac Lane, S. and Moerdijk, I. (1994), Sheaves in Geometry and Logic: A First Introduction to Topos Theory, Springer, Berlin.

Malament, D. B. (1977), 'The class of continuous timelike curves determines the topology of spacetime', Journal of Mathematical Physics 18(7), 1399-1404.

Malament, D. B. (2012), Topics in the Foundations of General Relativity and Newtonian Gravitation Theory, University of Chicago Press, Chicago.

Misner, C. W., Thorne, K. S. and Wheeler, J. A. (1973), Gravitation, W. H. Freeman, San Francisco.

Müller, T. (2013), 'A generalized manifold topology for branching space-times', Philosophy of Science 80(5), 1089-1100.

Nestruev, J. (2003), Smooth manifolds and observables, Springer, Berlin. 
North, J. (2009), 'The "structure” of physics: A case study', The Journal of Philosophy 106(2), 5788.

North, J. (2018), A new approach to the relational-substantival debate, in K. Bennett and D. W. Zimmerman, eds, 'Oxford Studies in Metaphysics', Vol. 11, Oxford University Press, Oxford, pp. 3-43.

Norton, J. D. (2008), 'Why constructive relativity fails', British Journal for the Philosophy of Science 59, 821-834.

Norton, J. D. (2015), The hole argument, in E. N. Zalta, ed., 'The Stanford Encyclopedia of Philosophy', Fall 2015 edn, Metaphysics Research Lab, Stanford University.

O’Neill, B. (1983), Semi-Riemannian Geometry, with Applications to Relativity, Academic Press, San Diego.

Penrose, R. (1968), Structure of space-time, in C. M. DeWitt and J. A. Wheeler, eds, 'Batelle Rencontres: 1967 Lectures in Mathematics and Physics', W. A. Benjamin, New York, pp. 121235.

Penrose, R. (1979), Singularities and time-asymmetry, in S. W. Hawking and W. Israel, eds, 'General Relativity: An Einstein Centenary Survey', Cambridge University Press, Cambridge, pp. 581-638.

Penrose, R. and Rindler, W. (1984), Spinors and Space-Time, Vol. 1, Cambridge University Press, Cambridge.

Pfister, H. and King, M. (2015), Inertia and Gravitation: The Fundamental Nature and Structure of Space-Time, Springer, Cham.

Placek, T. (2014), Branching for general relativists, in T. Müller, ed., 'Nuel Belnap on Indeterminism and Free Action', Springer, Cham, pp. 191-222.

Placek, T. and Belnap, N. (2012), 'Indeterminism is a modal notion: Branching spacetimes and Earman's pruning', Synthese 187(2), 441-469.

Pooley, O. (2006), Points, particles and structural realism, in D. Rickles, S. French and J. Saatsi, eds, 'The Structural Foundations of Quantum Gravity', Oxford University Press, pp. 83-120.

Pooley, O. (2013), Substantivalist and relationalist approaches to spacetime, in R. Batterman, ed., 'The Oxford Handbook of Philosophy of Physics', Oxford University Press, Oxford, pp. 522586.

Robb, A. A. (1914), A Theory of Time and Space, Cambridge University Press, Cambridge.

Robb, A. A. (1921), The Absolte Relations of Time and Space, Cambridge University Press, Cambridge.

Robb, A. A. (1936), Geometry of Time and Space, Cambridge University Press, Cambridge. 
Roeper, P. (1997), 'Region-based topology’, Journal of Philosophical Logic 26, 251-309.

Rosenstock, S., Barrett, T. W. and Weatherall, J. O. (2015), 'On Einstein algebras and relativistic spacetimes', Studies in History and Philosophy of Modern Physics 52, 309-316.

Russell, B. (1927), The Analysis of Matter, Kegan Paul, London.

Ryckman, T. (2004), The Reign of Relativity: Philosophy in Physics, 1915-1925, Oxford University Press, Oxford.

Rynasiewicz, R. (1992), 'Rings, holes and substantivalism: On the program of Leibniz algebras', Philosophy of Science 59(4), 572-589.

Saraykar, R. and Janardhan, S. (2016), 'Zeeman-like topologies in special and general theory of relativity', Journal of Modern Physics 7, 627-641.

Schild, A. (1967), Lectures on general relativity theory, in J. Ehlers, ed., 'Relativity Theory and Astrophysics', Vol. 1, American Mathematical Society, Providence, pp. 1-104.

Schmidt, B. G. (1973), 'Conditions on a connection to be a metric connection', Communications in Mathematical Physics 29(1), 55-59.

Sen, R. N. (2010), Causality, measurement theory and the differentiable structure of space-time, Cambridge University Press, Cambridge.

Simons, P. (1987), Parts: A Study in Ontology, Oxford University Press, Oxford.

Sklar, L. (1977), Facts, conventions, and assumptions in the theory of space-time, in J. Earman, C. Glymour and J. Stachel, eds, 'Foundations of Space-Time Theories', Vol. VIII of Minnesota Studies in the Philosophy of Science, University of Minnesota Press, Minneapolis, pp. 206-274.

Sładkowski, J. (2001), 'Gravity on exotic $\mathbb{R}^{4}$ with few symmetries', International Journal of Modern Physics D 10, 311-313.

Stachel, J. (1999), On the interpretation of the Einstein-Cartan formalism, in A. Harvey, ed., 'On Einstein's Path', Springer, New York, pp. 475-485.

Székely, G. (2015), What properties of numbers are needed to model accelerated observers in relativity?, in J.-Y. Beziau, D. Krause and J. B. Arenhart, eds, 'Conceptual Clarifications: Tributes to Patrick Suppes (1922-2014)’, College Publications, London, pp. 161-174.

Székely, G. and Madarász, J. X. (2013), 'Special relativity over the field of rational numbers', International Journal of Theoretical Physics 52(5), 1706-1718.

Tennison, B. R. (1975), Sheaf Theory, Cambridge University Press, Cambridge.

Torretti, R. (1996), Relativity and Geometry, corrected edn, Dover, New York.

Trautman, A. (1976), 'A classification of space-time structures', Reports on Mathematical Physics 10(3), 297-310. 
Trautman, A. (2006), Einstein-Cartan theory, in J.-P. Françoise, G. L. Naber and S. T. Tsou, eds, 'Encyclopedia of Mathematical Physics', Vol. 2, Elsevier, Oxford, pp. 189-195.

Trautman, A. (2012), 'Editorial note to: J. Ehlers, F. A. E. Pirani and A. Schild, The geometry of free fall and light propagation', General Relativity and Gravitation 44(6), 1581-1586.

Wald, R. M. (1984), General Relativity, University of Chicago Press, Chicago.

Weatherall, J. O. (2017), Categories and the foundations of classical field theories, in E. Landry, ed., 'Categories for the Working Philosopher', Oxford University Press, Oxford, pp. 329-348.

Weatherall, J. O. (2018), 'Regarding the 'hole argument", The British Journal for the Philosophy of Science 69(2), 329-350.

Weyl, H. (1921), 'Zur Infinitesimalgeometrie: Einordnung der projektiven und der konformen Auffassung', Nachrichten der Königlichen Gesellschaft der Wissenschaften zu Gttingen 1921, 99112.

Whitehead, A. N. (1919), An Enquiry Concerning the Principles of Natural Knowledge, Cambridge University Press, Cambridge.

Whitehead, A. N. (1920), The Concept of Nature, Cambridge University Press, Cambridge.

Whitehead, A. N. (1929), Process and Reality. An Essay in Cosmology, Free Press, New York.

Whitney, H. (1936), 'Differentiable manifolds', Annals of Mathematics 37(3), 645-680.

Zeeman, E. C. (1964), 'Causality implies the Lorentz group', Journal of Mathematical Physics 5(4), 490-493. 